\title{
Prime Focus Spectrograph (PFS) for the Subaru telescope: ongoing integration and future plans
}

\section{Naoyuki Tamura, Naruhisa Takato, Atsushi Shimono, Yuki Moritani, Kiyoto Yabe, et al.}

\begin{abstract}
Naoyuki Tamura, Naruhisa Takato, Atsushi Shimono, Yuki Moritani, Kiyoto Yabe, Yuki Ishizuka, Yukiko Kamata, Akitoshi Ueda, Hrand Aghazarian, Stephané Arnouts, Robert H. Barkhouser, Philippe Balard, Rudy Barette, Mohamed Belhadi, Jill A. Burnham, Neven Caplar, Michael A. Carr, Pierre-Yves Chabaud, Yin-Chang Chang, HsinYo Chen, Chueh-Yi Chou, You-Hua Chu, Judith G. Cohen, Rodorigo P. de Almeida, Antonio C. de Oliveira, Lígia S. de Oliveira, Richard G. Dekany, Kjetil Dohlen, Jesulino B. dos Santos, Leandro H. dos Santos, Richard S. Ellis, Maximilian Fabricius, Decio Ferreira, Hisanori Furusawa, Javier Garcia-Carpio, Mirek Golebiowski, Johannes Gross, James E. Gunn, Randolph Hammond, Albert Harding, Murdock Hart, Timothy M. Heckman, Paul T. P. Ho, Stephen C. Hope, David J. Hover, Shu-Fu Hsu, Yen-Shan Hu, Ping-Jie Huang, Sara Jamal, Marc Jaquet, Eric Jeschke, Yipeng Jing, Erin Kado-Fong, Jeniffer L. Karr, Masahiko Kimura, Matthew E. King, Michitaro Koike, Eiichiro Komatsu, Vincent Le Brun, Olivier Le Fèvre, Arnaud Le Fur, David Le Mignant, Hung-Hsu Ling, Craig P. Loomis, Robert H. Lupton, Fabrice Madec, Peter H. Mao, Danilo Marchesini, Lucas S. Marrara, Dmitry Medvedev, Sogo Mineo, Yosuke Minowa, Hitoshi Murayama, Graham J. Murray, Youichi Ohyama, Masato Onodera, Joseph Orndorff, Sandrine Pascal, Josh Peebles, Guillaume Pernot, Raphael Pourcelot, Daniel J. Reiley, Martin Reinecke, Mitsuko Roberts, Josimar A Rosa, Julien Rousselle, Alain Schmitt, Mark A. Schwochert, Micheal D.

Seiffert, Hassan Siddiqui, Stephen A. Smee, Laerte Sodré, Aaron J. Steinkraus, Michael A. Strauss, Christian

Surace, Philip J. Tait, Masahiro Takada, Tomonori Tamura, Masayuki Tanaka, Yoko Tanaka, Aniruddha R. Thakar, Orlando Verducci, Didier Vibert, Shiang-Yu Wang, Zuo Wang, Chih-Yi Wen, Suzanne Werner, Yoshihiko

Yamada, Chi-Hung Yan, Naoki Yasuda, Hiroshige Yoshida, Michitoshi Yoshida, "Prime Focus Spectrograph (PFS) for the Subaru telescope: ongoing integration and future plans," Proc. SPIE 10702, Ground-based and Airborne Instrumentation for Astronomy VII, 107021C (27 July 2018); doi: 10.1117/12.2311871
\end{abstract}

Event: SPIE Astronomical Telescopes + Instrumentation, 2018, Austin, Texas, United States 


\section{Prime Focus Spectrograph (PFS) for the Subaru telescope: Ongoing integration and future plans}

Naoyuki Tamura $^{\mathrm{a}}$, Naruhisa Takato $^{\mathrm{b}}$, Atsushi Shimono ${ }^{\mathrm{a}}$, Yuki Moritani ${ }^{\mathrm{a}}$, Kiyoto Yabe ${ }^{\mathrm{a}}$, Yuki Ishizuka $^{a}$, Yukiko Kamatac ${ }^{c}$, Akitoshi Uedac ${ }^{c}$,Hrand Aghazarian ${ }^{\text {, }}$, Stéphane Arnouts ${ }^{\mathrm{e}}$, Robert

H. Barkhouser ${ }^{\mathrm{f}}$, Philippe Balard ${ }^{\mathrm{e}}$, Rudy Barette ${ }^{\mathrm{e}}$, Mohamed Belhadi ${ }^{\mathrm{e}}$, Jill A. Burnham ${ }^{\mathrm{g}}$, Neven Caplar ${ }^{\mathrm{h}}$, Michael A. Carr ${ }^{\mathrm{h}}$, Pierre-Yves Chabaud ${ }^{\mathrm{e}}$, Yin-Chang Changi, Hsin-Yo Chen ${ }^{\mathrm{i}}$, Chueh-Yi Chou ${ }^{i}$, You-Hua Chu ${ }^{i}$, Judith G. Cohen ${ }^{g}$, Rodrigo P. de Almeida ${ }^{j}$, Antonio C. de Oliveira $^{j}$, Ligia S. de Oliveira ${ }^{j}$, Richard G. Dekany ${ }^{g}$, Kjetil Dohlen ${ }^{\mathrm{e}}$, Jesulino B. dos Santos $^{\mathrm{j}}$, Leandro H. dos Santos ${ }^{j}$, Richard S. Ellis ${ }^{k}$, Maximilian Fabricius ${ }^{1}$, Décio Ferreira ${ }^{j}$, Hisanori Furusawa $^{\text {, }}$, Javier Garciá-Carpio ${ }^{1}$, Mirek Golebiowskif ${ }^{f}$, Johannes Gross ${ }^{\mathrm{d}}$, James E. Gunn ${ }^{\mathrm{h}}$, Randolph Hammond ${ }^{f}$, Albert Harding ${ }^{f}$, Murdock Hart ${ }^{f}$, Timothy M. Heckman ${ }^{f}$, Paul T. P. Ho ${ }^{i}$, Stephen C. Hope ${ }^{\text {, }}$, David J. Hover ${ }^{g}$, Shu-Fu Hsu ${ }^{i}$, Yen-Shan Hu ${ }^{i}$, Ping-Jie Huangi, Sara Jamal $^{\mathrm{e}}$, Marc Jaquet ${ }^{\mathrm{e}}$, Eric Jeschke ${ }^{\mathrm{b}}$, Yipeng Jing ${ }^{\mathrm{m}}$, Erin Kado-Fong ${ }^{\mathrm{h}}$, Jennifer L. Karr ${ }^{\mathrm{i}}$, Masahiko Kimura ${ }^{i}$, Matthew E. King ${ }^{\mathrm{d}}$, Michitaro Koike ${ }^{\mathrm{c}}$, Eiichiro Komatsu ${ }^{\mathrm{a}, \mathrm{n}}$, Vincent Le Brun $^{\mathrm{e}}$, Olivier Le Févre ${ }^{\mathrm{e}}$, Arnaud Le Fur ${ }^{\mathrm{e}}$, David Le Mignant ${ }^{\mathrm{e}}$, Hung-Hsu Ling ${ }^{\mathrm{i}}$, Craig P. Loomis $^{h}$, Robert H. Lupton ${ }^{\text {, }}$ Fabrice Madec ${ }^{\mathrm{e}}$, Peter H. Mao ${ }^{\mathrm{d}, \mathrm{g}}$, Danilo Marchesini ${ }^{\mathrm{o}}$, Lucas S. Marrara $^{j}$, Dmitry Medvedev ${ }^{f}$, Sogo Mineo ${ }^{c}$, Yosuke Minowa ${ }^{b}$, Hitoshi Murayama ${ }^{a, p, q}$, Graham

J. Murray $^{\mathrm{r}}$, Youichi Ohyama ${ }^{\mathrm{i}}$, Masato Onodera ${ }^{\mathrm{b}}$, Joseph Orndorff ${ }^{\mathrm{f}}$, Sandrine Pascal ${ }^{\mathrm{e}}$, Josh Peebles $^{\mathrm{f}}$, Guillaume Pernot ${ }^{\mathrm{e}}$, Raphael Pourcelot ${ }^{\mathrm{e}}$, Daniel J. Reiley ${ }^{\mathrm{g}}$, Martin Reinecke ${ }^{\mathrm{n}}$, Mitsuko Roberts $^{\mathrm{g}}$, Josimar A. Rosa ${ }^{\mathrm{j}}$, Julien Rousselle ${ }^{\mathrm{b}}$, Alain Schmitt ${ }^{\mathrm{e}}$, Mark A. Schwochert ${ }^{\mathrm{d}}$, Michael D. Seiffert ${ }^{\mathrm{d}, \mathrm{g}}$, Hassan Siddiqui ${ }^{\mathrm{h}}$, Stephen A. Smee ${ }^{\mathrm{f}}$, Laerte Sodre Jr. ${ }^{\mathrm{s}}$, Aaron J. Steinkraus ${ }^{\mathrm{d}}$, Michael A. Strauss ${ }^{h}$, Christian Surace ${ }^{\mathrm{e}}$, Philip J. Tait ${ }^{\mathrm{b}}$, Masahiro Takada ${ }^{\mathrm{a}}$, Tomonori Tamura ${ }^{\mathrm{b}}$,

Masayuki Tanakac, Yoko Tanaka ${ }^{\mathrm{b}}$, Aniruddha R. Thakar ${ }^{\mathrm{f}}$, Orlando Verducci Jr. ${ }^{\mathrm{j}}$, Didier

Vibert $^{\mathrm{e}}$, Shiang-Yu Wang ${ }^{\mathrm{i}}$, Zuo Wang ${ }^{\mathrm{f}}$, Chih-Yi Wen ${ }^{\mathrm{i}}$, Suzanne Werner ${ }^{\mathrm{f}}$, Yoshihiko Yamada ${ }^{\mathrm{c}}$, Chi-Hung Yan ${ }^{\mathrm{i}}$, Naoki Yasuda ${ }^{\mathrm{a}}$, Hiroshige Yoshida ${ }^{\mathrm{b}}$, and Michitoshi Yoshida ${ }^{\mathrm{b}}$

${ }^{a}$ Kavli Institute for the Physics and Mathematics of the Universe (WPI), The University of Tokyo Institutes for Advanced Study, The University of Tokyo, Kashiwa, Chiba 277-8583, Japan

bSubaru Telescope, National Astronomical Observatory of Japan, 650 North A'ohoku Place, Hilo, HI 96720, USA

'National Astronomical Observatory of Japan, 2-21-1 Osawa, Mitaka, Tokyo 181-8588, Japan

d Jet Propulsion Laboratory, 4800 Oak Grove Dr., Pasadena, CA 91109, USA

eAix Marseille Université, CNRS, CNES, LAM (Laboratoire d'Astrophysique de Marseille) UMR 7326, 13388, Marseille, France

f Johns Hopkins University, Department of Physics and Astronomy, 3701 San Martin Drive, Baltimore, MD 21218, USA

${ }^{\mathrm{g}}$ California Institute of Technology, 1200 E California Blvd, Pasadena, CA 91125, USA

${ }^{\text {h}}$ Princeton University, Department of Astrophysical Sciences, Princeton, NJ 08544, USA

${ }^{i}$ Academia Sinica, Institute of Astronomy and Astrophysics, P. O. Box 23-141, Taipei, Taiwan

¡Laboratório Nacional de Astrofísica, Itajubá, 37504-364 Minas Gerais, Brazil

${ }^{k}$ Department of Physics and Astronomy, University College London, Gower Street, London, WC1E 6BT, UK

Ground-based and Airborne Instrumentation for Astronomy VII, edited by Christopher J. Evans, Luc Simard, Hideki Takami, Proc. of SPIE Vol. 10702, 107021C · @ 2018 SPIE · CCC code: 0277-786X/18/\$18 · doi: 10.1117/12.2311871 


\author{
${ }^{1}$ Max Planck Institute for Extraterrestrial Physics, Giessenbachstrasse, D-85748 Garching, \\ Germany \\ ${ }^{\mathrm{m}}$ Center for Astronomy and Astrophysics, Department of Physics and Astronomy, Shanghai \\ Jiao Tong University, Shanghai 200240, China \\ ${ }^{\mathrm{n}}$ Max-Planck-Institut für Astrophysik, Karl-Schwarzschild Str. 1, D-85741 Garching, Germany \\ ${ }^{\circ}$ Tufts University, Physics \& Astronomy Department, Collaborative Learning and Innovation \\ Complex (CLIC), 574 Boston Avenue, Medford, MA 02155, USA \\ ${ }^{\mathrm{P} U n i v e r s i t y ~ o f ~ C a l i f o r n i a, ~ B e r k e l e y, ~ C A ~ 94720, ~ U S A ~}$ \\ ${ }^{q}$ Lawrence Berkeley National Laboratory, MS 50A-5104, Berkeley, CA 94720, USA \\ ${ }^{\mathrm{r}}$ Centre for Advanced Instrumentation, Durham University, South Road, Durham, DH1 3LE, \\ UK \\ sDepartamento de Astronomia, Instituto de Astronomia, Geofísica e Ciências Atmosféricas, \\ Universidade de São Paulo, Rua do Matão 1226, Cidade Universitária, 05508-090 São Paulo, \\ Brazil
}

\begin{abstract}
PFS (Prime Focus Spectrograph), a next generation facility instrument on the 8.2-meter Subaru Telescope, is a very wide-field, massively multiplexed, optical and near-infrared spectrograph. Exploiting the Subaru prime focus, 2394 reconfigurable fibers will be distributed over the $1.3 \mathrm{deg}$ field of view. The spectrograph has been designed with 3 arms of blue, red, and near-infrared cameras to simultaneously observe spectra from $380 \mathrm{~nm}$ to $1260 \mathrm{~nm}$ in one exposure at a resolution of $\sim 1.6-2.7 \AA$. An international collaboration is developing this instrument under the initiative of Kavli IPMU. The project recently started undertaking the commissioning process of a subsystem at the Subaru Telescope side, with the integration and test processes of the other subsystems ongoing in parallel. We are aiming to start engineering night-sky operations in 2019, and observations for scientific use in 2021. This article gives an overview of the instrument, current project status and future paths forward.
\end{abstract}

Keywords: Subaru Telescope, future instrument, wide-field instrument, multi-object spectroscopy, optical and near-infrared spectroscopy, optical spectroscopy, near-infrared spectroscopy, international collaboration, optical fibers

\title{
1. INTRODUCTION
}

The wide-field capability at the prime focus is clearly one of the key advantages of the $8.2 \mathrm{~m}$ Subaru Telescope, and has been exploited to deliver valuable scientific data. After the previous generation instruments (Suprime$\mathrm{Cam}^{1}$ and FMOS, ${ }^{23}$ ), Hyper Suprime-Cam (HSC) ${ }^{4}$ has been in quite successful operation. In particular, HSC is a very wide-field imager with a 1.5-degree diameter field of view "paved" by $1162 \mathrm{~K} \times 4 \mathrm{~K}$ CCDs, and since 2014 , a 5-year, 300-night survey program is on-going in the framework of Subaru Strategic Program (SSP). Exciting scientific results are being published continuously and rapidly: Over 100 science papers have been published to date, and a special issue of Publication of Astronomical Society of Japan (PASJ) in January 2018 included 40 papers on first results from the HSC survey.

PFS (Prime Focus Spectrograph), as described in this article, is a very wide-field, massively multiplexed, optical and near-infrared (NIR) spectrometer. The focal plane will be equipped with 2394 reconfigurable fibers distributed in the 1.3-degree wide hexagonal field of view. The spectrograph has been designed to cover a wide range of wavelengths simultaneously from $380 \mathrm{~nm}$ to $1260 \mathrm{~nm}$ in one exposure. The PFS and HSC instrumentation projects are under the umbrella of the Subaru Measurement of Images and Redshifts (SuMIRe) project (PI: H. Murayama) aiming to conduct deep and wide sky surveys exploiting the unique capability of the Subaru Telescope. It should be emphasized that HSC and PFS enable deep imaging and spectroscopic surveys of the

Further author information: (Send correspondence to Naoyuki Tamura.)

E-mail: naoyuki.tamura@ipmu.jp, Telephone: +81 (0)4 71366531 
same patches of the sky using the same $8.2 \mathrm{~m}$ telescope, allowing one to have good understandings of various systematics in the data.

Envisioning a large survey in the SSP framework, the PFS science team has developed a preliminary survey plan and has developed top-level requirements for the instrument. ${ }^{5}$ The goal is to address key questions in three main fields: cosmology, galaxy \& AGN evolution, and Galactic archaeology, and from the joint implications, to understand the dark sector of the universe. The team has been continuously updating and refining the plan in details, as the instrument characteristics, technical constraints and software components on the survey observation process are better understood. The combination of the wide field, high multiplicity, and high number density of the fibers on the focal plane offers an opportunity of designing a unique survey on these three core science cases envisioned in the PFS SSP survey.

The development of this instrument has been undertaken by an international collaboration at the initiative of Kavli IPMU, with work packages for subsystem and subcomponent development assigned to various collaborating institutions. The project is now in the phase of construction, integration and test aiming to start operations for scientific use in 2021. In parallel, detailed modeling of the instrument and output spectral images are on-going in order to characterize the instrument on-sky capabilities and accordingly finalize the SSP survey design. This way, we aim to conduct commissioning observations efficiently and start a PFS SSP program in a timely manner subsequently. In what follows, the instrument basics are described in $\S 2$, and then updates of a few major aspects of the project are presented in $\S 3$. This article is summarized and a timeline for the future developments is given in $\S 4$.

\section{THE INSTRUMENT}

The PFS instrument is composed of four subsystems, whose distribution on the telescope is illustrated in Fig. 1: The lights from astronomical objects and sky are fed to the fibers configured at the Subaru prime focus, are then transmitted via the fiber cable to the spectrograph system in the telescope enclosure building, and the spectral images of them are delivered on the spectrograph detectors. We here give an overview of these subsystems.

At the Subaru prime focus, HSC has already been in science operation with the wide field of view and the reasonably flat focal plane provided by the new Wide-Field Corrector lens system (WFC). The WFC will be used for PFS as well. Mechanically, the new prime focus housing unit "POpt2" is integrated with WFC and accommodates the HSC instrument inside. When PFS is in operation, the HSC instrument will be taken out and our Prime Focus Instrument (PFI) will be installed in POpt2.

PFI has been developed by the collaboration of $\mathrm{CIT}^{*} \&$ NASA JPL ${ }^{\dagger}, \mathrm{LNA}^{\ddagger}$, and ASIAA ${ }^{\S}$, accommodating key subcomponents such as the fiber positioner system, science \& fiducial fiber system, Acquisition \& Guide (AG) cameras, and calibration system. The fiber positioner system consists of 42 modules each of which accommodates 57 "Cobra" rotary actuators populated with science fibers. The tip of each science fiber is equipped with a plano-concave microlens to increase the focal ratio of the input beam to the fiber to $2.8{ }^{6}$ The Cobra engineering model actuators have been assembled to a prototype module and tested. The results show satisfactory target convergence performance in the patrol field of each fiber. ${ }^{7}$ These subcomponents will be integrated into PFI and be fully tested at ASIAA before the delivery to Subaru. ${ }^{8}$

Metrology Camera System (MCS) developed by ASIAA ${ }^{9}$ is installed at the Cassegrain focus of the telescope. Because the fiber positioners have no encoders, an external system is required to drive them to the proper position. MCS corresponds to this external system which takes images of the science and fiducial fibers back-lit from the other side of prime focus, and then measures the fiber positions, enabling closed-loop operation of the positioners. MCS is capable of taking an image of all the back-lit science and fiducial fibers on the prime focus in one exposure. The $380 \mathrm{~mm}$-diameter aperture system is designed to minimize the impacts of the dome seeing effect and small-scale figure errors of the WFC lens surface shapes.

\footnotetext{
${ }^{*}$ California Institute of Technology

${ }^{\dagger}$ Jet Propulsion Laboratory

${ }^{\ddagger}$ Laboratório Nacional de Astrofísica (Brazil)

${ }^{\S}$ Academia Sinica Institute of Astronomy and Astrophysics (Taiwan)
} 


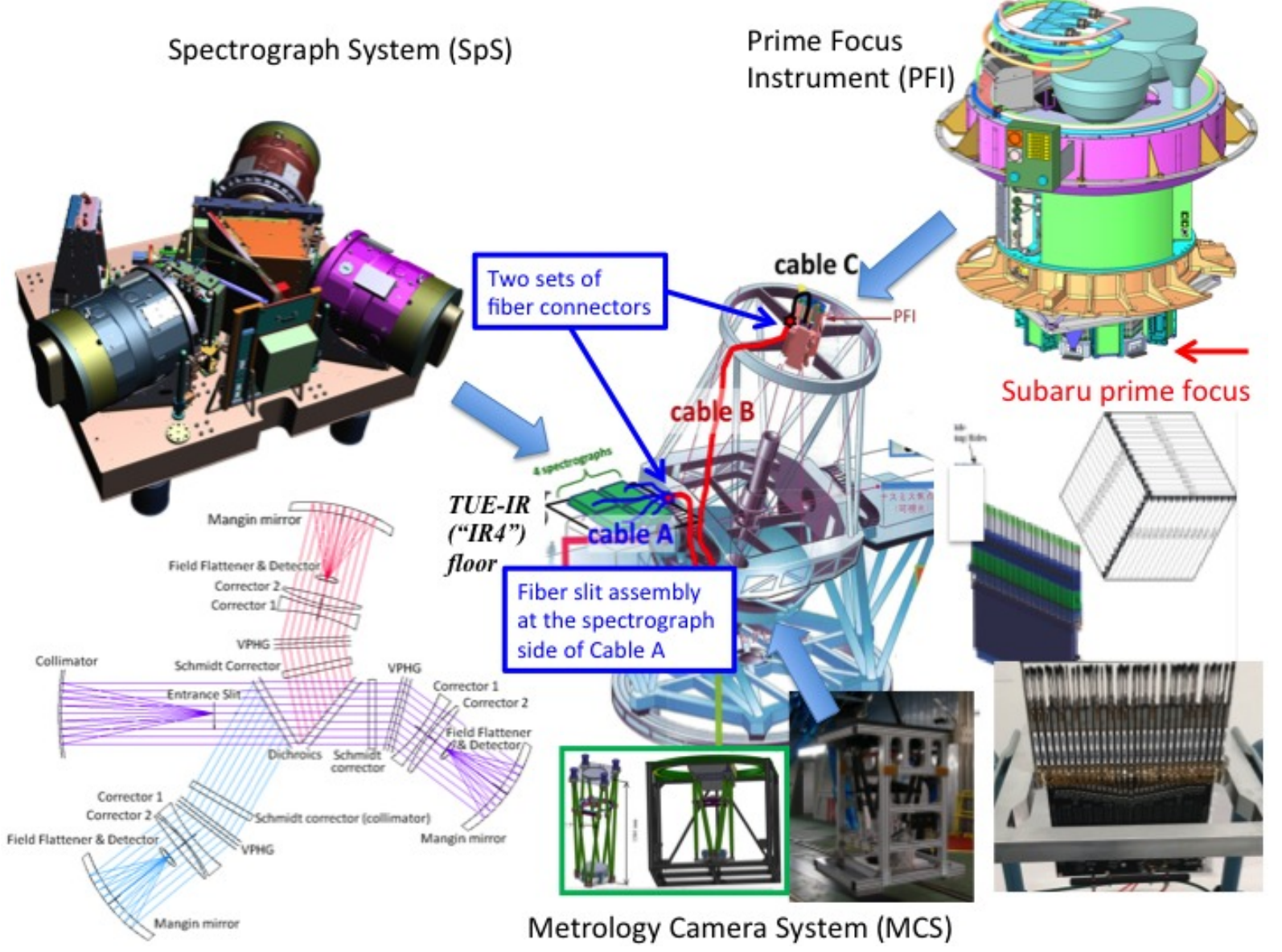

Figure 1. A schematic view of the configuration of PFS subsystems. An overall sketch of the Subaru Telescope is presented in the middle with the PFS fiber cable routed from the prime focus to the spectrograph system. On the right, a solid model of PFI (top), a schematic view of the focal plane (middle), and a photo of the Cobra fiber positioner module are presented. On the left, a solid model of one spectrograph module (top) and its ray-trace view (bottom) are shown. At the bottom center, a photo of MCS in the Subaru Telescope dome and its solid models are presented next to each other. 
Spectrograph System (SpS) is being integrated at LAM ${ }^{\uparrow},{ }^{10}$ with the fiber system delivered by LNA and the camera dewars \& detectors developed by Princeton University $(\mathrm{PU})^{11}$ and Johns Hopkins University (JHU). ${ }^{12-14}$ The divergent beams from the science fibers on the pseudo slit are collimated and then split into blue, red and NIR channels by two dichroic mirrors. After this, the beam is dispersed by the VPH grating and spectral images are formed on the detectors. A grating exchange mechanism allows a higher dispersion VPH grism to be accommodated in the system and deliver medium resolution spectra in the red channel with no changes in the other parts of SpS. SpS consists of four spectrograph modules (SM) each of which is identically designed to deliver $\sim 600$ spectral images on the detectors.

Fiber system "FOCCoS" to be delivered by LNA ${ }^{15-18}$ consists of three parts: Two short-fiber systems accommodated in PFI and SpS, and a long cable system that is routed on the telescope to connect PFI and SpS. These three parts are connected together by two sets of fiber connectors. One is needed at the telescope top end to make POpt2 detachable from the telescope, and the other is in front of SpS to ease the delivery and integration of SpS at Subaru and to make the operation and maintenance activities independent of the other PFS subsystems.

In Table 1, the major instrument parameters are listed. While the basic parameters are fixed, one should refer to the PFS official web site http://pfs.ipmu.jp/ for the fiber reconfiguration time, the throughput and the estimated on-sky sensitivity and related details as they will be updated as the instrument is built, integrated and tested and the characteristics are better understood.

Table 1. PFS major instrument parameters

\begin{tabular}{|c|c|c|c|c|}
\hline \multicolumn{5}{|c|}{ Prime Focus Instrument (PFI) } \\
\hline Field of view (hexagonal) & \multicolumn{4}{|c|}{$\begin{array}{c}\text { Diameter of circumscribed circle: } 1.38 \mathrm{deg} \\
\text { Area: } 1.25 \mathrm{deg}^{2}\end{array}$} \\
\hline Number of fibers & \multicolumn{4}{|c|}{2394 science fibers and 96 fixed fiducial fibers. } \\
\hline Fiber density & \multicolumn{4}{|c|}{$2000 \mathrm{deg}^{-2}\left(0.6 \operatorname{arcmin}^{-2}\right)$} \\
\hline Fiber core diameter & \multicolumn{4}{|c|}{$127 \mu \mathrm{m}(=1.12(1.02)$ arcsec at the field center (edge), respectively) } \\
\hline Positioner pitch & \multicolumn{4}{|c|}{$8 \mathrm{~mm}(=90.4(82.4)$ arcsec at the field center (edge), respectively) } \\
\hline Positioner patrol field diameter & \multicolumn{4}{|c|}{$9.5 \mathrm{~mm}(=107.4(97.9)$ arcsec at the field center (edge), respectively) } \\
\hline Fiber minimum separation & \multicolumn{4}{|c|}{$\sim 30 \operatorname{arcsec}$} \\
\hline Fiber configuration time & \multicolumn{4}{|c|}{$\sim 60-120 \mathrm{sec}(\mathrm{TBC})$} \\
\hline Number of AG cameras & \multicolumn{4}{|c|}{6} \\
\hline Field of view per AG camera & \multicolumn{4}{|c|}{$5.1 \operatorname{arcmin}^{2}$} \\
\hline Sensitivity of AG camera & \multicolumn{4}{|c|}{$S / N=30(100)$ for $r=20 \mathrm{mag}(\mathrm{AB}), 1(10)$ sec exposure. } \\
\hline \multicolumn{5}{|c|}{ Spectrograph System (SpS) } \\
\hline \multirow{2}{*}{ Spectral arms } & \multirow{2}{*}{ Blue } & \multicolumn{2}{|c|}{ Red } & \multirow{2}{*}{ NIR } \\
\hline & & Low Res. & Mid. Res. & \\
\hline Spectral coverage & $380-650 \mathrm{~nm}$ & 630-970nm & $710-885 \mathrm{~nm}$ & $940-1260 \mathrm{~nm}$ \\
\hline Dispersion & $0.7 \AA /$ pix & $0.9 \AA / \mathrm{pix}$ & $0.4 \AA /$ pix & $0.8 \AA /$ pix \\
\hline Spectral resolution & $2.1 \AA$ & $2.7 \AA$ & $1.6 \AA$ & $2.4 \AA$ \\
\hline Resolving power & 2300 & 3000 & 5000 & 4300 \\
\hline SpS throughput & $53 \%($ at $500 \mathrm{~nm})$ & $57 \%($ at $800 \mathrm{~nm})$ & $54 \%($ at $800 \mathrm{~nm})$ & $33 \%$ (at $1100 \mathrm{~nm})$ \\
\hline
\end{tabular}

\section{RECENT DEVELOPMENTS}

In this section, some of the major updates during the last two years after the previous article ${ }^{19}$ are highlighted. Since the other articles cover the current statuses and updates of the developments of FOCCoS, ${ }^{16},{ }^{17},{ }^{18} \mathrm{SpS},{ }^{10},{ }^{14}$ and MCS, ${ }^{9}$ this article forcuses on other updates.

\footnotetext{
"Laboratoire d'Astrophysique de Marseille
} 


\subsection{The Collaboration}

In November 2016, MPE ${ }^{\|}$in Germany joined the PFS collaboration. Then MPA, which had been in the collaboration since 2014, and MPE formed a participating group to be a full member. Recently, in April 2018, a group of North-East US universities** consisting of 7 senior scientists led by Danilo Marcesini (Tufts) joined the PFS collaboration as a partial member. We believe these new partners have made the collaboration even stronger and further reinforced the chance of success of instrument development and survey science. There are still occasional contacts from potential candidates for new partners with which the PFS steering committee is negotiating.

\subsubsection{MCS Commissioning}

Details are described in the other article ${ }^{9}$ so a short summary is presented here. MCS was fully integrated and tested at ASIAA by March 2018. After the PFS project and the Subaru Telescope observatory jointly performed a formal pre-shipping review meeting, MCS was delivered to the Subaru Telescope Hilo base facility on April 20. From the week of April 23, the members from ASIAA and Kavli IPMU then reassembled MCS in the lab in the Hilo base with supports from the observatory, coarsely checked the optical alignment with a green laser, and confirmed the system's basic functions such as camera operation, shutter operation, and telemetry. After another formal review meeting to make sure the progress of the work at the Hilo base and the readiness of the subsequent work, MCS was transported to the Subaru Telescope facility on the summit of Maunakea on May 1.

The commissioning works continued at the Subaru summit. Firstly MCS was reassembled again for detailed optical alignment. This time an additional optics was integrated to make the system achromatic. After the success of image quality confirmation, MCS was integrated with the Cassegrain container on May 31 and was attached to the telescope on June 8 (see Fig. 2). We then successfully confirmed various interfaces to the telescope system and infrastructure and operated the on-telescope MCS.

In advance of and in parallel to the MCS commissioning, the commissioning of PFS software system is undertaken at the observatory site. In orchestrating the operation of PFS subsystems in coordination with the telescope system, the key software component is Messaging Hub System (MHS): As has been demonstrated in the SDSS operations at Apache Point Observatory, it efficiently organizes distributed processes providing uniform communication interfaces between software components. This in fact eases the integration process. While each subsystem is tested and validated with MHS at the development site, MHS is implemented and commissioned as a part of the PFS software system at the observatory site and engages communications with the Subaru side. Then, when a subsystem is delivered with its control software, it can immediately be hooked up with this MHS at the observatory for testing and operation. In advance of the MCS delivery, the team implemented the PFS servers and infrastructures at the observatory and commissioned MHS and a few other software components needed at the PFS system level. MCS is therefore the first subsystem delivered to the observatory not only as the hardware itself but also from the viewpoint of software integration, so its commissioning is an important milestone for the project.

\subsection{Fiber Positioner System and Prime Focus Instrument}

The production of the fiber positioner "Cobra" was completed and all of them were delivered. Now the integration of 57 Cobras to so-called "Cobra module" is under way at CIT. Once a Cobra module is fully integrated, it is installed on a test cube and firstly the basic parameters of each Cobra such as the center of its patrol field and the radius of the circle drawn by the fiber when each of the two motors is rotated are measured. Then a set of targets are given to the Cobras to see how the fibers are moved by the Cobras accurately to the requested positions through an iterative process with a camera system to take images of backlit fibers. As was confirmed for the engineering module, ${ }^{7}$ the production modules that have been integrated so far also show good target convergence: We evaluate the performance in terms of a signal-to-noise $(\mathrm{S} / \mathrm{N})$ ratio (i.e. the performance is considered to be better as the residual distance of a fiber from the target position gets smaller more quickly), and typically $\mathrm{S} / \mathrm{N}$ is maximized after $\sim 6-7$ iterations. ${ }^{19}$ In addition to these tests, the fiber aiming angles and the focus positions of the fibers are also measured on this test cube.

\footnotetext{
"Max Planck Institute for Extraterrestrial Physics

** The member institutes are: Tufts University, Columbia University, University of Conneticut, the University of Illinois at Urbana-Campaign, and University of Pittsburgh.
} 


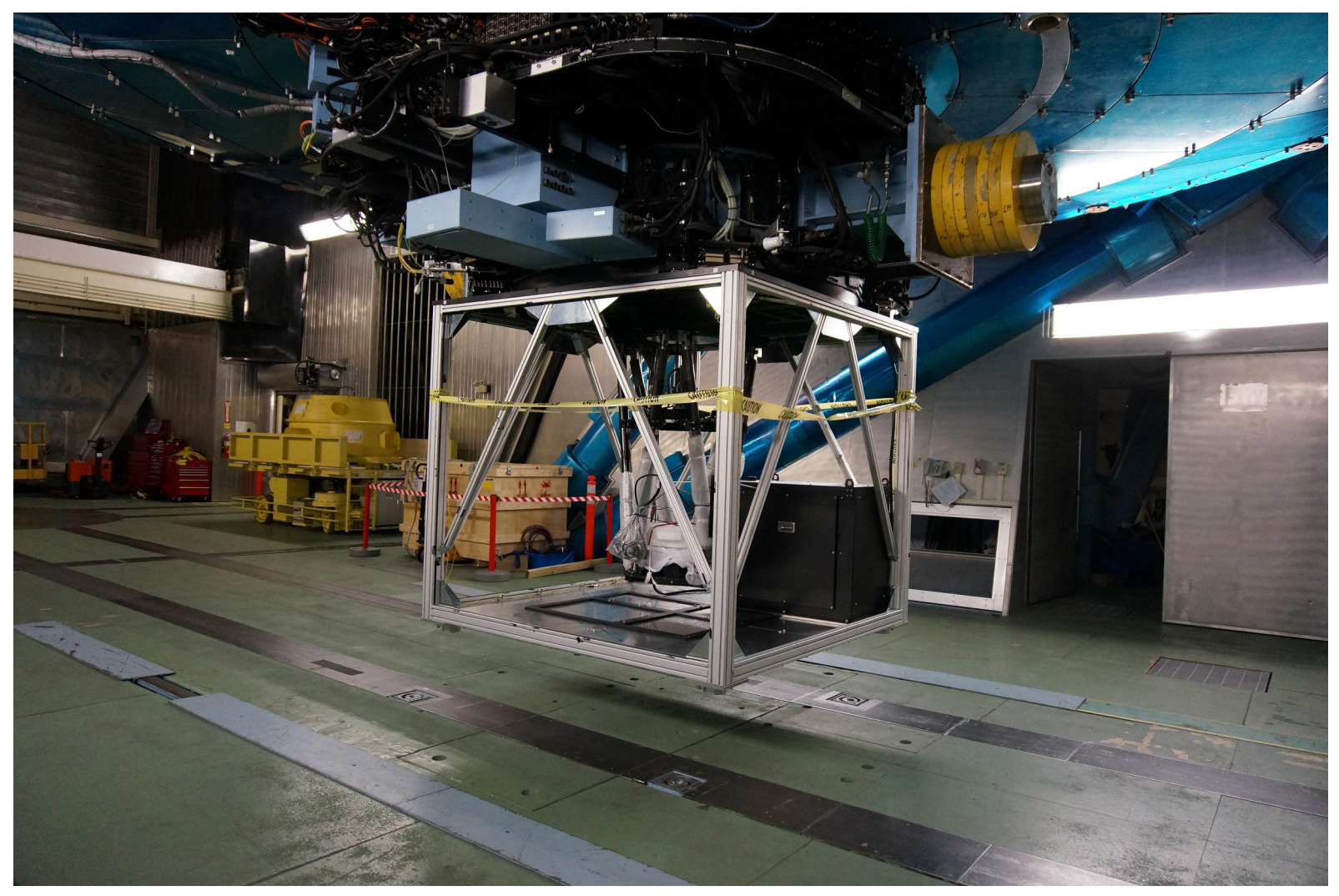

Figure 2. The Metrology Camera System (MCS) installed to the Cassegrain focus of the Subaru Telescope.

Once all such measurements are done, a module is packed for shipping, and is delivered to Taiwan. After receiving inspections and basic tests to confirm the Cobras function as expected, the module will be installed into the PFI mechanical structure. The major components (Fig. 3) are now under integration and the characterization of the assembly such as deflection measurement as a function of tilt is also under way on a test stand in a temperature-controlled room. ${ }^{8}$ Soon the first Cobra module will be installed and, using the metrology system on a calibrated XY scanner on the test stand, detailed testing of the module will be started. The software development is progressing in parallel. Although some hardware devices and optical configuration are different, the system developed on the test stand is to mimic the operation of the Cobra modules on PFI and MCS on the telescope. This way, we will validate PFI, in particular the fiber positioner system, in such a way that we can minimize commissioning processes after PFI is delivered to the Subaru telescope.

\subsection{Subaru Infrastructure for PFS Spectrograph System}

To install the SpS of PFS, the "infrared-side" 4th floor in the telescope enclosure building was refurbished with reinforcements to stably support all the weight as the $\mathrm{SpS}$ requires. On top of this upgraded floor, a Spectrograph Clean Room (SCR) has been built (Fig. 4) to accommodate the four SMs inside with a crane for integration activities, while the electronics racks and a few auxiliary components are installed outside SCR. Currently cabling and piping works are ongoing, and tests of monitoring and controling the environment will be started next.

\subsection{Prototype On-Telescope Fiber Cable}

In April 2017, the prototype of the on-telescope fiber cable (so-called "Cable B" 18 which will be routed on the telescope to optically connect PFI and SpS was manufactured. The structure of the cable is the following: ${ }^{20}$ The fibers are distributed into segmented polymer tubes and these tubes are stranded around a central strength member. In this way, any tensile stress experienced by the cable is carried by the strength member, not the 

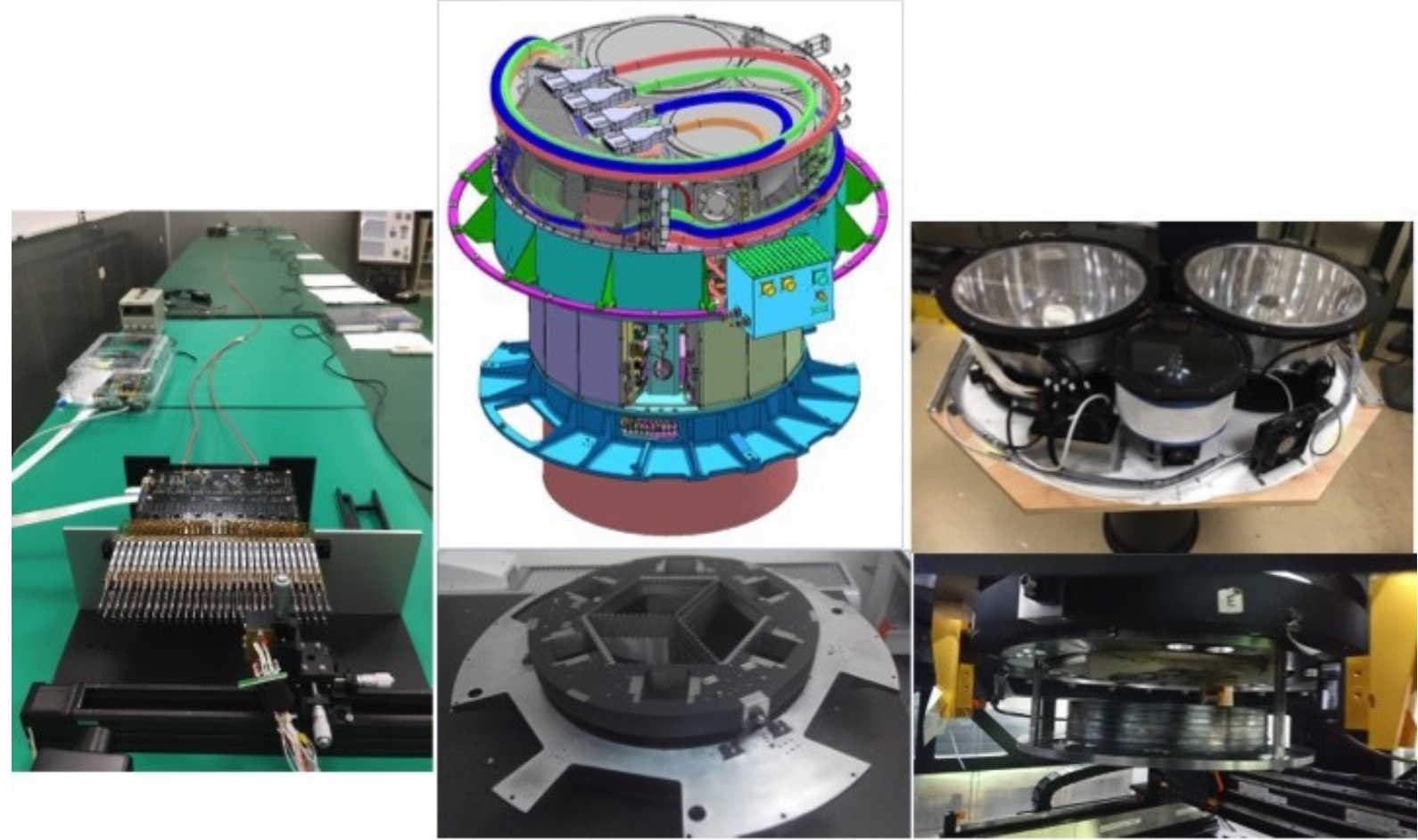

Figure 3. (Upper middle) 3D model of Prime Focus Instrument. (Left) A Cobra module under testing on the bench. (Lower middle) Hexagonal focal plane on the Cobra Optical Bench (COB) integrated with Positioner Frame (PF). (Lower right) The assembly of $\mathrm{COB}$ and $\mathrm{PF}$ under deflection testing. (Upper right) Calibration lamp system to be integrated on top of PFI.
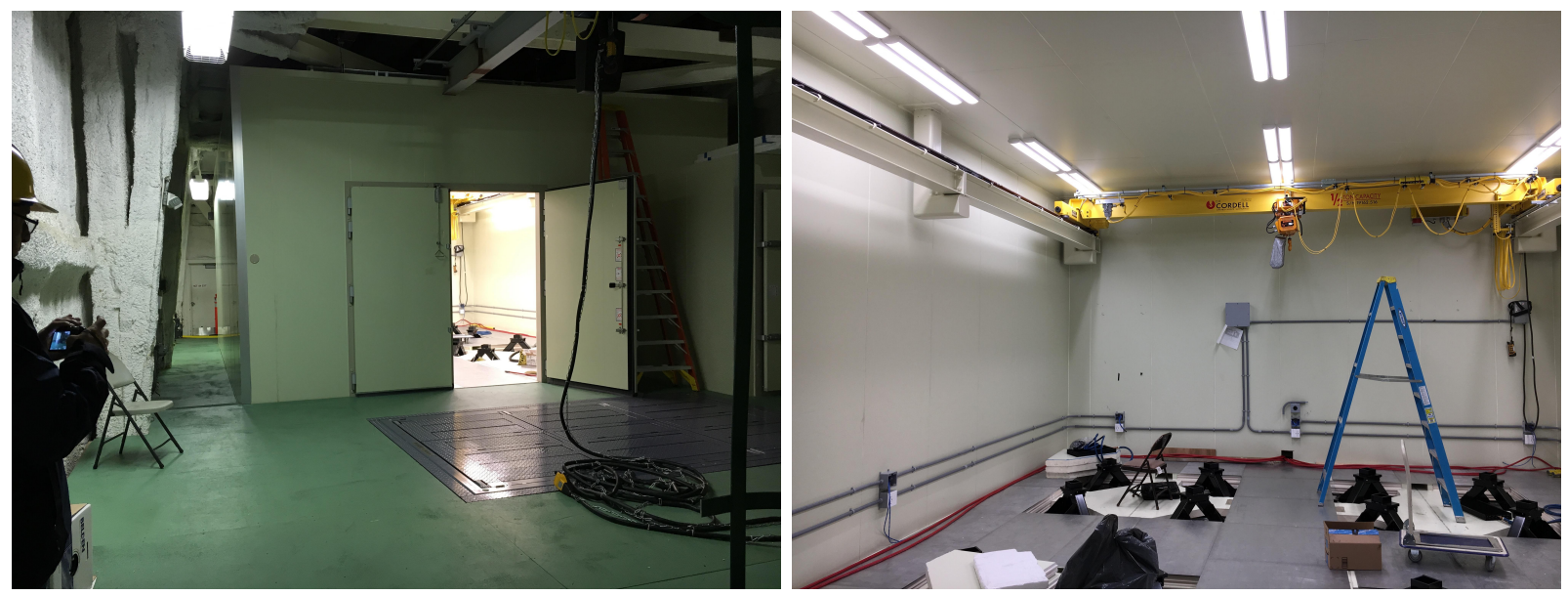

Figure 4. Photos of the Spectrograph Clean Room (SCR): (Left) Outside, and (right) inside.

fibers. This stranded assembly is then wrapped by a vinyl tape and is inserted into a PVC-coated flexible metal conduit. The conduit protects the cable against radial stresses (crushing forces) and the risk of environmental contamination such as water or oil leaks. We then carried out the measurement of Focal Ratio Degradation (FRD) measurement of a sample of the fibers in the cable using a collimated beam technique, and we see no clear evidence for degradation of FRD due to the stranding.

Then recently, in January 2018, the prototype cable was installed on the telescope (Figure 5). The prototype 


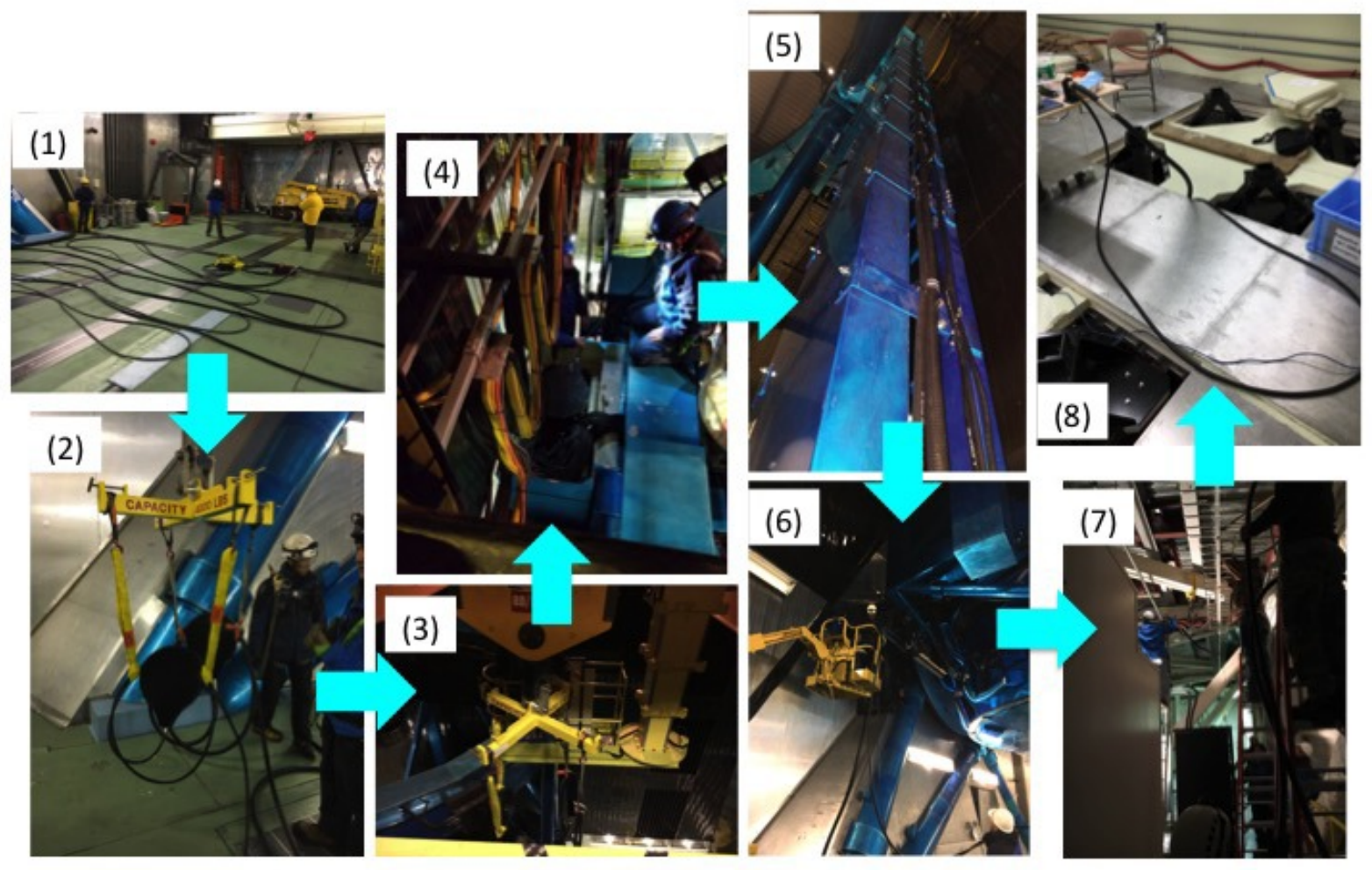

Figure 5. Following the arrows, the sequence of the work to install the prototype fiber cable onto the telescope is presented: (1) The fiber cable is loosely routed on the floor in the telescope area. (2) \& (3) Its one end is lifted by the crane to the telescope top end. (4) the cable is installed on the telescope top-end. (5) The cable is routed down along the ladder at the side of the telescope structure. (6) The other end of the cable is drawn into the telescope enclosure building through the clearance near the telescope elevation axis. (7) The cable is further routed inside the enclosure building. (8) The cable is fully routed up to the clean room for the spectrograph system.

does not range entirely as expected by the real cable, but is routed from the telescope top-end outer ring to SCR for SpS. After the installation, we monitored the FRD variation of one fiber in the cable as we gave local changes to the cable route and changed the telescope elevation angle. We will continue such tests again in the future to optimize the details of the fiber route and installation method, and also to quantify the FRD variation as a function of telescope elevation angle over a substantial period of time.

\subsection{Survey Simulation and Optimization}

As mentioned previously in 1, the PFS science team is aiming at an PFS SSP survey accommodating multiple components, ${ }^{5}{ }^{19}$ The expected survey period is substantially long (up to 360 nights over $\sim 5$ years), the target fields are distributed over a wide range of sky, the candidates for spectroscopic follow-up observation with PFS can be heterogeneous in terms of type, magnitude, redshift, etc in particular in the case of galaxy-AGN evolution component. It is therefore crucial to simulate the entire survey process and optimize details. These simulative studies are being conducted under the initiative of the team at Kavli IPMU.

In the course of the survey observation, fiber-to-object allocation is one of the key processes. It needs to be optimized under various requirements such as the number and distribution of sky fibers for good data calibration and accurate sky subtraction, and also restrictions from the instrument such as avoidance of collisions between 
adjacent Cobras. In addition, at a given exposure in the middle of the survey, the optimization should be carried out with the progress of the survey by that time and the planned exposures in the rest of the survey taken into account: While the goal is to maximize the amount of scientific outputs at the end of the survey, it can be accomplished not necessarily by optimizing the fiber allocation independently on an individual exposure. The fact that we are still in the middle of many things such as instrument characterization and calibration, and detailed survey planning where studies and discussions are ongoing about how to prioritize different classes of objects and how to prioritize different survey components. So an algorithm for fiber-to-object allocation needs to be fairly flexible so that it can be optimized with various requirements and constraints, and metrics of evaluating scientific values. The team at MPA and MPE are working on this development employing the network flow apporach (e.g. ${ }^{21}$ ) and a prototype is about to be released for the science team, while the other software product based on a simpler optimization strategy has been developed by the same team and used for various simulative studies so far.

\subsection{HSC+PFS Science Database}

One of the unique strengths in the PFS SSP survey is the fact that the survey can be planned based on the HSC data. The HSC images are deep and sharp, so they allow us to cleanly select suitable targets for follow-up spectroscopy. Also, PFS spectra will be merged for analyses on top of detailed studies with HSC images and photometry, and this combination will be very powerful. This indicates in other words that it is crucial to have a science database with HSC images and PFS spectra both integrated and therefore scientists will be allowed to efficiently access to both. The data from both HSC SSP survey and PFS SSP survey must have a lot of legacy value, and needless to say such a database will be essential for archival data science, but also the database can be important as a tool in the middle of PFS SSP survey for data validation and survey progress monitoring.

The development of HSC+PFS science database is ongoing by the collaboration of the teams at National Astronomical Observatory of Japan (NAOJ) and JHU. Recently they have developed a prototype database for trials and evaluations by the scientists in the PFS collaboration. Through their feedbacks, the team will fully capture required functions and features and find rooms for improvements of the system. We plan to make several iterations in the next few years to have the system ready for science by the time we start the PFS survey.

\section{SUMMARY AND FUTURE PERSPECTIVES}

PFS (Prime Focus Spectrograph), a next generation facility instrument on the Subaru Telescope, is a very wide-field, massively multiplexed, optical and NIR spectrograph: The prime focus will be equipped with 2394 reconfigurable fibers in the $1.3 \mathrm{deg}$ field of view, and the spectra simultaneously cover the wide range of wavelengths from $380 \mathrm{~nm}$ to $1260 \mathrm{~nm}$ at one exposure. The development of this instrument by an international collaboration under the initiative of Kavli IPMU is finalizing the design and starting the construction at the subsystem level. We expect the subsystems to be integrated and validated by the collaborators and be delivered to the telescope site in 2018-2019. We will then carry out system integration and start engineering observations in 2019. Based on our preliminary plans, $\sim 1.5$ years will be necessary to complete engineering observations (including a certain period for optimization and stabilization of the performance and operation), so we expect to start science operation and a 5-year PFS SSP survey in 2021. The science team is developing a detailed survey strategy to be refined in the next two years, and the technical team is committing to this by brushing up the estimates of on-sky instrument sensitivity and carrying out survey and data simulations. Information on the instrument development and survey strategy will be posted and updated on the PFS official website http://pfs.ipmu.jp/. In addition, news, events and milestones are reported in the PFS official blog http://pfs.ipmu.jp/blog/.

PFS and HSC, a unique set of powerful survey instruments exploiting the prime focus of the Subaru Telescope, will be crucial strategic pieces for the Subaru Telescope through the 2020's into the 2030's: The 6.5m effectiveaperture Large Synoptic Survey Telescope (LSST) is a US-led project starting around 2020 and is currently under construction, and will enable an ultimate dedicated imaging survey of the universe. However, a spectroscopic follow-up observation of objects found by LSST objects is not yet planned. The extremely large-aperture telescope such as the Thirty-Meter Telescope (TMT) in which the Japanese community is participating is aiming to start its operation after 2025, but has a small field-of-view, and much better suits for a detailed spectroscopic observation of very interesting and/or rare astronomical objects. TMT is therefore complementary to PFS in light of their 
roles, meaning that there must be good chances of synergy. From a similar viewpoint, a synergy with a space telescope is exciting, and there are some provisional discussions toward a collaboration to combine data from WFIRST and PFS. Accordingly, PFS on the Subaru Telescope will still be a world-leading astronomical facility not only in 2020s but also in 2030s to further advance our understanding of the physics of the Universe

\section{ACKNOWLEDGMENTS}

We appreciate all the contributions from the PFS science team to the instrument requirements definitions and the survey planning. We also thank all the people involved with this PFS project in the past in various formats. Without their efforts and contributions, the project would not even start or continue to exist until now. We are grateful to the staffs (in addition to those on the author list) at National Astronomical Observatory of Japan and the Subaru Telescope observatory for their contributions to the development of the PFS instrument, the modifications of the telescope system and other infrastructures to accept PFS, the preparations of PFS system integration and engineering observations, and various other aspects such as the administrative supports. Our thanks should also be sent to the staffs at Durham University in the United Kingdom, for their supports of the development of the PFS fiber system as the consultancy. We gratefully acknowledge support from the Funding Program for World-Leading Innovative R\&D on Science and Technology (FIRST) program "Subaru Measurements of Images and Redshifts (SuMIRe)", CSTP, Japan. This work is supported by JSPS KAKENHI Grant Numbers JP15H05893, JP15K21733, and JP15H05892. The work in ASIAA, Taiwan, is supported by the Academia Sinica of Taiwan. The work in Brazil is supported by the FAPESP grant 2012/00800-4.

\section{REFERENCES}

[1] Miyazaki, S., Komiyama, Y., Sekiguchi, M., Okamura, S., Doi, M., Furusawa, H., Hamabe, M., Imi, K., Kimura, M., Nakata, F., Okada, N., Ouchi, M., Shimasaku, K., Yagi, M., and Yasuda, N., "Subaru Prime Focus Camera - Suprime-Cam," PASJ 54, 833-853 (2002).

[2] Kimura, M., Maihara, T., Iwamuro, F., Akiyama, M., and Tamura, N. et al., "Fibre Multi-Object Spectrograph (FMOS) for the Subaru Telescope," PASJ 62, 1135-1147 (2010).

[3] Tamura, N., Takato, N., Iwamuro, F., Akiyama, M., and Kimura, M. et al., "Subaru FMOS now and future," in [Ground-based and Airborne Instrumentation for Astronomy IV], Ian S. McLean, Suzanne K. Ramsay, H. T., ed., Proc. SPIE 8446, 8446M0 (2012).

[4] Miyazaki, S., Komiyama, Y., Nakaya, H., Kamata, Y., and Doi, Y. et al., "Hyper Suprime Cam," in [Ground-based Instrumentation for Astronomy], Moorwood, A. F. M. and Masanori, I., eds., Proc. SPIE 5492, 1228-1242 (2012).

[5] Takada, M., Ellis, R. S., Chiba, M., Greene, J. E., and Aihara, H. et al., "Extragalactic science, cosmology, and galactic archaeology with the Subaru Prime Focus Spectrograph," PASJ 66(1), id.R1 (2014).

[6] Takato, N., Tanaka, Y., Gunn, J. E., Tamura, N., and Shimono, A. et al., "Design and performance of a F/\#-conversion microlens for prime focus spectrograph at Subaru Telescope," in [Ground-based and Airborne Instrumentation for Astronomy V], Suzanne K. Ramsay, Ian S. McLean, H. T., ed., Proc. SPIE 9147, 914765 (2014).

[7] Fisher, C., Morantz, C., Braun, D., Seiffert, M., and Aghazarian, H. et al., "Developing engineering model cobra fiber positioners for the Subaru Telescope's prime focus spectrometer," in [Advances in Optical and Mechanical Technologies for Telescopes and Instrumentation], Ramón Navarro, Colin R. Cunningham, A. A. B., ed., Proc. SPIE 9151, 91511Y (2014).

[8] Wang, S.-Y., Schwochert, M. A., Huang, P.-J., Chen, H.-Y., and Kimura, M. et al., "The current status of prime focus instrument of Subaru prime focus spectrograph," in [Ground-based and Airborne Instrumentation for Astronomy VI], Christopher J. Evans, Luc Simard, H. T., ed., Proc. SPIE 9908, id. 9908829 pp. (2016).

[9] Wang, S.-Y., Chou, R. C. Y., Huang, P.-J., Chang, Y.-C., and Ling, H.-S. et al., "Metrology camera system of prime focus spectrograph for Subaru telescope," in [Ground-based and Airborne Instrumentation for Astronomy VII], Christopher J. Evans, Luc Simard, H. T., ed., Proc. SPIE 10702 (2018). 
[10] Madec, F., Pascal, S., Le Fur, A., Le Mignant, D., and Dohlen, K. et al., "SUBARU prime focus spectrograph integration and performance at lam," in [Ground-based and Airborne Instrumentation for Astronomy VII], Christopher J. Evans, Luc Simard, H. T., ed., Proc. SPIE 10702 (2018).

[11] Gunn, J. E., Fitzgerald, R., Hart, M., Hope, S. C., Loomis, C., Peacock, G. O., Golebiowski, M., Carr, M., Smee, S. A., Tamura, N., Shimono, A., and Takato, N., "Detector and control system design and performance for the SuMIRe Prime Focus Spectrograph (PFS) cameras," in [Ground-based and Airborne Instrumentation for Astronomy VI], Christopher J. Evans, Luc Simard, H. T., ed., Proc. SPIE 9908 (2016).

[12] Smee, S. A., Gunn, J. E., Golebiowski, M., Hope, S. C., Madec, F., Gabriel, J.-F., Loomis, C., Le fur, A., Dohlen, K., Le Mignant, D., Barkhouser, R., Carr, M. A., Hart, M., Tamura, N., Shimono, A., and Takato, N., "Visible camera cryostat design and performance for the SuMIRe Prime Focus Spectrograph (PFS)," in [Ground-based and Airborne Instrumentation for Astronomy VI], Christopher J. Evans, Luc Simard, H. T., ed., Proc. SPIE 9908-333 (2016).

[13] Hart, M., Barkhouser, R. H., Gunn, J. E., and Smee, S. A., "A novel reflectometer for relative reflectance measurements of ccds," in [High Energy, Optical, and Infrared Detectors for Astronomy VII], Andrew D. Holland, J. B., ed., Proc. SPIE 9915, id. 99152D 6 pp. (2016).

[14] Hart, M., Barkhouser, R. H., Gunn, J. E., and Smee, S. A., "Focal plane array alignment and cryogenic surface topography measurements for the Prime Focus Spectrograph," in [High Energy, Optical, and Infrared Detectors for Astronomy VIII], Andrew D. Holland, J. B., ed., Proc. SPIE 10709 (2018).

[15] de Oliveira, A. C., de Oliveira, L. S., de Arruda, M. V., Souza Marrara, L., and dos Santos, L. H. et al., "Fiber optical cable and connector system (FOCCoS) for PFS/Subaru," in [Advances in Optical and Mechanical Technologies for Telescopes and Instrumentation], Ramón Navarro, Colin R. Cunningham, A. A. B., ed., Proc. SPIE 9151, 91514G (2014).

[16] de Oliveira, A. C. et al., "Fiber input assembly for PFS/Subaru," in [Ground-based and Airborne Instrumentation for Astronomy VII], Christopher J. Evans, Luc Simard, H. T., ed., Proc. SPIE 10702 (2018).

[17] de Oliveira, A. C., de Oliveira, L. S., Gunn, J. E., Ferreira, D., and Moritani, Y. et al., "Slit device assembly of Prime Focus Spectrograph for Subaru telescope," in [Ground-based and Airborne Instrumentation for Astronomy VII], Christopher J. Evans, Luc Simard, H. T., ed., Proc. SPIE 10702 (2018).

[18] de Oliveira, A. C., de Oliveira, L. S., Ferreira, D., Souza Marrara, L., and dos Santos, L. H. et al., "Permanent optical fiber cable for Prime Focus Spectrograph and Subaru telescope cable b," in [Ground-based and Airborne Instrumentation for Astronomy VII], Christopher J. Evans, Luc Simard, H. T., ed., Proc. SPIE 10702 (2018).

[19] Tamura, N., Takato, N., Shimono, A., Moritani, Y., and Yabe, K. et al., "Prime Focus Spectrograph (PFS) for the Subaru telescope: overview, recent progress, and future perspectives," in [Ground-based and Airborne Instrumentation for Astronomy VI], Christopher J. Evans, Luc Simard, H. T., ed., Proc. SPIE 9908, id. 99081M 17 pp. (2016).

[20] Murray, G. J., Tamura, N., Takato, N., Ekpenyong, P., and Jenkins, D. et al., "An optimal method for producing low-stress fibre optic cables for astronomy," in [Astronomical Optics: Design, Manufacture, and Test of Space and Ground Systems], Tony B. Hull, Dae Wook Kim, P. H., ed., Proc. SPIE 10401 (2017).

[21] Blanton, M. R., Lin, H., Lupton, R. H., Maley, F. M., Young, N., Zehavi, I., and Loveday, J., "An efficient targeting strategy for multiobject spectrograph surveys: the sloan digital sky survey tiling algorithm," $A J$ 125, Issue 4, pp. 2276-2286 (2003). 